\title{
Hypokalaemic Paralysis - A double trouble from concurrent Thyrotoxicosis and Gitelman syndrome: A report of two cases
}

\author{
Chandrika Jayakanthi Subasinghe ${ }^{1}$, Sonali Sihindi Chapa Gunatilake ${ }^{1}$, Nirmala Dushyanthi Sirisena ${ }^{2}$, Knut Erik Berge ${ }^{3}$, \\ Trond Paul Leren ${ }^{3}$, Uditha Bulugahapitiya ${ }^{1}$,Vajira Harshadeva Weerabaddana Dissanayake ${ }^{2}$ \\ ${ }^{1}$ Endocrinology Unit, Colombo South Teaching Hospital, Kalubowila, Sri Lanka \\ ${ }^{2}$ Human Genetics Unit, Faculty of Medicine, University of Colombo, Colombo 08, Sri Lanka \\ ${ }^{3}$ Unit for Cardiac and Cardiovascular Genetics, Department for Medical Genetics, Oslo University Hospital, Ullevaal, Oslo, Norway
}

Abstract

\section{Background}

Hypokalaemic paralysis is a rare group of disorders with concomitant muscle weakness and hypokalaemia. Thyrotoxicosis is a recognized and a common cause for hypokalaemic paralysis among Asians, in which intracellular shift of potassium is enhanced. Gitelman syndrome, an autosomal recessive renal salt loosing condition is characterized by hypokalaemia, hypocalciuria and hypomagnesaemia. Concurrence of these two conditions is very rarely reported.

\section{Case Presentation}

We report two genetically unrelated Sri Lankan patients who presented with concurrent thyrotoxicosis and Gitelman syndrome. Both of them presented with symptomatic hypokalaemia and detected to have thyrotoxicosis. One had Grave's disease, while the other patient had toxic multinodular goiter. They were initially managed symptomatically for thyrotoxic hypokalaemic paralysis. Despite rendering euthyroidism with medical management, they persisted to have symtomatic hypokalaemia. On evaluation for a second pathology, we detected them to have Gitelman syndrome, which is a rare concurrence.

\section{Conclusion}

Elevated thyroid hormone levels can precipitate a paralytic episode in a patient with subclinical chronic hypokalaemia due to an additional underlying disease. Gitelman syndrome is such a disease which should be considered and actively looked for in a patient with persistent hypokalaemia following rendering euthyroidism.

Key words: Thyrotoxicosis, Gitelman syndrome, Hypokalaemic paralysis, Case report

Correspondence e mail: chandrika.ucfm@gmail.com

ORCID ID: https://orcid.org/0000-0002-4454-0194

Copyright: This is an open-access article distributed under the terms of the Creative Commons Attribution License, which permits unrestricted use, distribution, and reproduction in any medium, provided the original author and source are credited (CC BY 4.0)

\section{Background}

Hypokalaemic paralysis is transient flaccid neuromuscular weakness of varying intensity with associated hypokalaemia. It is a rare group of disorders with a prevalence of 1 in 100,000 (1). Hypokalaemic paralysis is classified as primary (familial) and secondary. Primary hypokalaemic paralysis, which is the common form, is characterized by autosomal dominant inheritance of channelopathies. Secondary hypokalaemic paralysis has an identifiable cause such as thyrotoxicosis, primary hyperaldosteronism and renal or gastrointestinal potassium loosing conditions (2).
Thyrotoxic hypokalaemic paralysis (THP) is a lifethreatening complication of thyrotoxicosis which is commonly seen among Asian descend. Okinaka et al. reported the prevalence of THP as $1.8 \%$ among Japanese thyrotoxic patients and $8 \%$ among Japanese thyrotoxic male patients, indicating a male predominance ${ }^{(3,4)}$. Despite the higher incidence of thyrotoxicosis among females, male to female ratio of THP ranges from 17:1 to 70:1 (5). Grave's disease is commonly associated with THP but any cause of thyrotoxicosis can precipitate hypokalaemic paralysis (6-10). 
Thyroid hormones induce $\mathrm{Na} / \mathrm{K}$ ATPase activity in cells, thus shifting potassium from extracellular to intracellular compartment, resulting in hypokalaemia (5). There are two forms of THP, familial form in which there is a mutation in the KCNE3 gene encoding voltage gated potassium channel and sporadic form, where there is activation of $\mathrm{Na} / \mathrm{K}$ ATPase pump due to high thyroxin levels. It is reported that $25-30 \%$ of patients with THP do not exhibit features of thyrotoxicosis $(6,11)$, however, euthyroid patients are free from spontaneous or induced attacks (5,12). Yet, finding of thyrotoxicosis and hypokalaemic paralysis does not always make the definitive diagnosis of THP as patient may have another underlying condition leading to chronic hypokalaemia which increases the patients' vulnerability for paralytic episodes (diuretic abuse, laxative abuse, hyperaldosteronism and conditions with renal or gastrointestinal potassium loss such as Gitelman syndrome, Bartters' syndrome, chronic diarrhea) (14).

Gitelman syndrome(GS) is a rare autosomal recessive renal salt wasting disorder in which there is an inactivation mutation of gene SCL12A3 which encodes the thiazide sensitive sodium-chloride co-transporter in the distal convoluting tubule ${ }^{(15)}$. It causes renal loss of potassium due to secondary hyperaldosteonism and is characterized by combination of hypokalaemia, hypomagnesaemia, hypocalciuria and metabolic alkalosis. Patients present in their adolescence or adult life with the spectrum of symptoms ranging from muscle weakness to hypokalaemic paralysis. Presence of concurrent thyrotoxicosis and Gitelman syndrome is rare and extensive literature survey revealed only few case reports ${ }^{(16-20)}$. Both conditions result in hypokalaemia, which further precipitate muscle paralysis. Herewith we present two genetically unrelated patients from Sri Lanka who presented with thyrotoxicosis with accompanied Gitelman syndrome.

\section{Case Presentation}

In our Endocrinology unit, we have cared for two genetically unrelated patients with concurrent thyrotoxicosis and Gitelman syndrome, who presented with hypokalaemia within 2 years duration, which is a very rare occurrence. Patient 01 is a 32year-old unmarried Sri Lankan male who was initially presented with loss of weight, malaise, body aches and pains, muscle weakness and easy fatigability. On evaluation of these nonspecific symptoms, he was found to be thyrotoxic and hypokalaemic (Table 1). He was diagnosed to have Grave's thyrotoxicosis as evidenced by mild diffuse enlargement of thyroid ultrasonically and high Thyroid Receptor antibody (TRAb) titre. THP was considered as the diagnosis for muscle weakness associated with hypokalaemia in this thyrotoxic young male on clinical grounds. He was rendered euthyroid with a titration regimen of carbimazole within 6 months. Despite being euthyroid and being on potassium supplements, it was difficult to achieve normal serum potassium levels and he persisted to have muscle fatigue. Due to lack of treatment response, an underlying second cause for hypokalaemia was looked for. Further evaluation revealed the patient to be having normal blood pressure with hypokalaemic metabolic alkalosis, urinary potassium wasting associated with hypocalciuria and hypomagnesaemia (Table 1). Further genetic testing confirmed that he is homozygous for a mutation in SLC12A3 gene(Table 1), which has been previously reported in patients with Gitelman syndrome. He achieved remission of Grave's disease at 18 months and hypokalaemia was improved with aldosterone antagonism(spironolactone), potassium and magnesium supplements.

Patient 02 is a 48 -year-old mother of two, who was initially treated for toxic multi nodular goiter. While on titrating doses of carbimazole for 8 weeks, she presented with acute lower limb paralysis caused by severe hypokalaemia (Table 1). She was managed symptomatically with potassium correction with the clinical diagnosis of THP. Even though she recovered from initial paralytic episode, she persistently had hypokalaemia and muscle symptoms despite euthyroid status maintained with carbimazole, which was an unusual feature in THP. Further evaluation revealed the biochemical diagnosis of concurrent Gitelman syndrome with hypokalaemic metabolic alkalosis, hypocalciuria, hypomagnesemia, and normal blood pressure (Table 1). This patient was also positive for the same mutation in SLC12A3 gene which confirmed the biochemical diagnosis. Her hypokalaemic symptoms improved with spironolactone, potassium and magnesium supplements and she awaits definitive therapy for toxic nodular goiter with radio iodine therapy. Further genetic evaluation for familial thyrotoxic hypokalaemic paralysis was not performed due to lack of facilities.

Although two hypokalaemia producing mechanisms were operating simultaneously in these two patients, their symptoms were no worse than symptoms of THP or Gitelman syndrome. Rather patient 01 had very mild muscle symptoms, which was comparable with previously reported cases (16). No genetic association has been described between potassium channelopathy in familial THP and $\mathrm{Na}-\mathrm{Cl}$ transporter defect in Gitelman syndrome. But it is known that the intracellular shift of potassium occurred in the presence of elevated thyroid hormone levels could precipitate severe symptomatic hypokalaemia in subclinical Gitelman syndrome, which is a late onset disease in majority ${ }^{(14)}$. Extensive literature search revealed only five such reported cases of concurrent thyrotoxicosis and Gitelman syndrome (16-20). The association must be rare despite the possibility of concurrence, rather than been under reported, due to the fact that any patient with persistent hypokalaemia is usually screened for both thyrotoxicosis and renal potassium wasting under usual hypokalaemia evaluation protocols (21). On the other hand, very mild cases of Gitelman syndrome may be missed on evaluation because genetic testing is not freely available in all settings. 


\section{Table 1: Investigations summary of the two patients}

\begin{tabular}{|c|c|c|c|c|c|c|}
\hline \multirow[t]{2}{*}{ Patient } & \multicolumn{6}{|c|}{ Investigations on initial presentation } \\
\hline & $\begin{array}{c}\text { TSH } \\
(\mathrm{mIu} / \mathrm{l}) \\
(\mathrm{NL}-0.4-4.0)\end{array}$ & $\begin{array}{c}\text { FT4 } \\
(\mathrm{ng} / \mathrm{dl}) \\
(\mathrm{NL}-0.89-1.7)\end{array}$ & $\begin{array}{c}\mathrm{K} \\
(\mathrm{mmol} / \mathrm{l}) \\
(\mathrm{NL}-3.5-4.5)\end{array}$ & $\begin{array}{c}\mathrm{Mg} \\
(\mathrm{mmol} / \mathrm{l}) \\
(\mathrm{NL}-1.6-2.5)\end{array}$ & $\begin{array}{c}\text { Urinary } \mathrm{Ca} / \mathrm{Cr} \\
\text { ratio } \\
(\mathrm{NL}-0.1-0.58)\end{array}$ & $\begin{array}{l}\text { SLC12A3 } \\
\text { gene } \\
\text { mutation }\end{array}$ \\
\hline 01 & 0.012 & 2.45 & 2.1 & 1.12 & 0.02 & $\begin{array}{c}\text { p.A523T } \\
\text { C.1567G }>\text { A }\end{array}$ \\
\hline 02 & $<0.001$ & 5.79 & 1.6 & 0.8 & 0.01 & $\begin{array}{c}\text { p.A523T } \\
\text { C. } 1567 \mathrm{G}>\mathrm{A}\end{array}$ \\
\hline
\end{tabular}

\section{Conclusion}

We have reported two genetically unrelated Sri Lankan patients with concurrent dual pathologies for hypokalaemia. Thyrotoxicosis of any cause associated intracellular potassium shift could have precipitated hypokalaemia in these patients with subclinical Gitelman syndrome. Hypokalaemia is a life threatening emergency and its cause should be carefully looked for. In Asian region with high reported prevalence of THP, there is a natural tendency to conclude the diagnosis of THP in a hypokalaemic patient with thyrotoxicosis. But caution should be exercised and the possibility of thyrotoxicosis being the precipitant of symptoms in the presence of another underlying hypokalaemic disorder should be also entertained. Such patients should be monitored for recovery of hypokalaemia during euthyroidism. Persistent hypokalaemia despite rendering euthyroidism should be carefully evaluated for a concurrent pathology. Gitelman syndrome is one of possible pathologies, which should be actively looked for in such cases.

\section{List of abbreviations}

THP: Thyrotoxic HypokalaemicParalysis

GS: Gitelman syndrome

TRAb : Thyroid Receptor Antibody

Declarations

Ethics approval and consent to participate

Written informed consent was obtained from the patients for genetic testing as part of standard care. A copy of the written consent is available for review by the Editor of this journal.

\section{Consent for publication}

Written informed consent was obtained from the patients for the publication of all personal information contained in this case report. A copy of the written consent is available for review by the Editor of this journal.Competing
interestsThe authors declare that they have no competing interests.

\section{Availability of data and materials}

The primers and conditions for thermal cycling, and the datasets used and/or analysed during the current study are available from the corresponding author on reasonable request.

\section{Funding}

Not applicable

Authors' contributions CJS and SG drafted the manuscript. CJS, SG and UB clinically evaluated and managed the patients medically. NDS and VHWD coordinated molecular genetic testing and were involved in the review of the manuscript. KEB and TPL carried out the molecular genetic testing. All authors critically revised the final manuscript for important intellectual content and approved it.

Authors' information CJS (MBBS, MD, MRCP) and Sonali Gunatilake (MBBS, MD) are Senior registrars in Endocrinology. UB (MD, FRCP, FCCP, FACE) is a Consultant Endocrinologist. NDS (MBBS, MSc) is a Senior Lecturer/Clinical Geneticist. VHWD (MBBS, PhD) is a Professor and Medical Geneticist. KEB (MD, $\mathrm{PhD}$ ) is a Senior Researcher/Clinical Geneticist. TPL (MD, PhD) is Director at Unit for Cardiac and Cardiovascular Genetics, Oslo University Hospital.

\section{Acknowledgments}

We wish to express our gratitude to the patients, who kindly gave consent for their cases to be published in this paper. 
1. Fontaine B, Vale-Santos J, Jurkat-Rott K, Reboul J, Plassart E, Rime CS, Elbaz A, Heine R, Guimaraes J, Weissenbach J, et al. Mapping of the hypokalaemic periodic paralysis (HypoPP) locus to chromosome 1q31-32 in three European families. Nat Genet. 1994;6:267-272.

2. Arya SN. Periodic paralysis. JLACM 2002; 3(4): 374-82

3. Okinaka S, Shizume K, Iino S, Watanabe A, Irie M, Noguchi A, etal. The association of periodic paralysis and hyperthyroidism in Japan. J Clin Endocrinol Metab. 1957;17:1454-1459.

4. Shizume K, Shishiba Y, Kuma K, Noguchi S, Tajiri J, Ito K, etal. Comparison of the incidence of association of periodic paralysis and hyperthyroidism in Japan in 1957 and 1991. Endocrinol Jpn. 1992;39:315-318.

5. Kung AW. Clinical review. Thyrotoxic periodic paralysis: a diagnostic challenge. J Clin Endocrinol Metab. 2006;91:2490-2495.

6. Kelley DE, Gharib H, Kennedy FP, Duda RJ, McManis MB. Thyrotoxic periodic paralysis. Report of 10 cases and review of electromyographic findings. Arch Intern Med. 1989;149:2597-2600.

7. Tinker TD, Vannatta JB. Thyrotoxic hypokalemic periodic paralysis: report of four cases and review of the literature. J Okla State Med Assoc 1987;80:76-83.

8. Hsu FS, Tsai WS, Chau T, Chen HH, Chen YC, Lin SH. Thyrotopin-secreting pituitary adenoma presenting as hypokalemic periodic paralysis. Am J Med Sci. 2003;325:48-50.

9. Okihiro MM, Nordyke RA. Hypokalemic periodic paralysis: experimental precipitation with sodium liothyronine. JAMA. 1996;198:949-951.

10. Tran HA. Inadvertent iodine excess causing thyrotoxic hypokalemic periodic paralysis. Arch Intern Med. 2005;165:2536.

11. Kelley DE, Gharib H, Kennedy FP, Duda RJ, Jr, McManis PG. Thyrotoxic periodic paralysis. Report of 10 cases and review of electromyographic findings. Arch Intern Med. 1989;149:2597-600.

12. Ko GT, Chow CC, Yeung VT, Chan HH, Li JK, Cockram CS. Thyrotoxic periodic paralysis in a Chinese population. QJM. 1996;89:463-8.

13. Lin SH, Lin YF, Chen DT, Chu P, Hsu CW, Halperin ML. Laboratory tests to determine the cause of hypokalaemia and paralysis. Arch Intern Med. 2004;164:1561-1566. doi: 10.1001/archinte.164.14.1561.

14. Lin S, Huang C. Mechanism of thyrotoxic periodic paralysis. J Am Soc Nephrol. 2012; 23(6): 985-988.

15. Knoers NV, de Jong JC, Meij IC, Van Den Heuvel LP, Bindels RJ. Genetic renal disorders with hypomagnesemia and hypocalciuria. J Nephrol. 2003;16:293-6.

16. Baldane S, Ipekci SH, Celik S, Gundogdu A, Kebapcilar L. Hypokalemic paralysis due to thyrotoxicosis accompanied by Gitelman's syndrome. Indian J Nephrol. 2015; 25(2): 103-105.

17. Imashuku S, Teramura-Ikeda T, Kudo N, Kaneda S, Tajima T. Concurrence of thyrotoxicosis and Gitelman's syndrome-associated hypokalemia-induced periodic paralysis. Pediatr Rep. 2012; 4(2): e18.

18. Zha B, Zheng P, Liu J, Huang X. Coexistence of Graves' Disease in a 14-year-old young girl with Gitelman Syndrome. Clinical Endocrinology. 2015; 83(6): 995-7.

19. Suzuki A, Voshida M, Miura V, Oiso V. Gitelman's syndrome with silent thyroiditis. Nagoya J. Med. Sci. 2006 ;68: 63-65.

20. Hui D, Yan-hua L, Ze-ping S, Lin LI, Le-ping S. Coexistence of Gitelman's syndrome and thyroid disease: SLC12A3 gene analysis in two patients. Chinese Journal of Endocrinology and Metabolism. 2010; 26(5): 395-398.

21. Palmer BF. A physiologic based approach to the evaluation of a patient with hypokalaemia. Am J Kidney Dis. 2010; 56(6): 1184-90. 